\title{
UAS Remote Sensing Products for Supporting Extraction Management and Restoration Monitoring in Open-Pit Mines ${ }^{+}$
}

\author{
Vicenç Carabassa ${ }^{1, *}$, Pau Montero ${ }^{2}$, Marc Crespo ${ }^{2}$, Joan Cristian Padró ${ }^{2}$, Jaume Balagué ${ }^{3}$, \\ Josep Maria Alcañiz ${ }^{1}$, Lluís Brotons ${ }^{3}$ and Xavier Pons ${ }^{2}$ \\ 1 CREAF, E08193 Bellaterra (Cerdanyola del Vallès), Spain; josemaria.alcaniz@uab.es \\ 2 Grumets Research Group; Dep. Geografia, Edifici B, Universitat Autònoma de Barcelona, \\ 08193 Bellaterra, Spain; paumontero87@gmail.com (P.M.); marccrespotorres@gmail.com (M.C.); \\ joancristian.padro@uab.cat (J.C.P.); xavier.pons@uab.cat (X.P.) \\ 3 Unitat Mixta InForest (CTFC-CREAF), 25280 Solsona, Spain; jaume.balague@terradron.cat (J.B.); \\ lluis.brotons@ctfc.cat (L.B.) \\ * Correspondence: v.carabassa@creaf.uab.cat \\ † Presented at the TERRAenVISION 2019, Barcelona, Spain, 2-7 September 2019. \\ Published: 31 October 2019
}

\begin{abstract}
Accurate mapping of open-pit mine areas is a prerequisite for the efficient resource management of extractive companies, but also detailed mapping is a requirement for public administrations, especially regarding the monitoring of restored areas. In previous works, our team has contributed to a better knowledge of the use of Unmanned Aerial Systems (UAS) technologies for soil/vegetation restoration monitoring purposes, and in this work, we present a novel protocol to support combined interests of both private companies and governmental agencies. We introduce a case study in which we show the capability of multispectral sensors onboard of a low-weight multicopter to describe land cover typologies in restored areas (such as grass, scrubs, trees, topsoil and mine spoils) by applying remote sensing and GIS techniques. Moreover, we assess the capability of digital terrain models (Digital Elevation Model, Digital Surface Model, Digital Slope Model) derived from photogrammetric techniques, to provide useful and fast topographic information for the proper management of open-pit mine exploitation and restoration. By applying these techniques, we present a cost-effective workflow adequate to monitor land cover dynamics in restored areas, but also volumetric changes in stockpiles, waste dumps and extraction faces. This combined approach, supporting both environmental and industrial needs, aims to enhance the collaboration between sectors, establishing synergies, reducing costs by sharing knowledge, and adding transparency to their relation.
\end{abstract}

Keywords: drone; multispectral imagery; RGB imagery; morphometric; mapping; vegetation classification

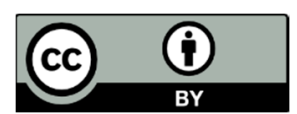

(C) 2019 by the authors. Licensee MDPI, Basel, Switzerland. This article is an open access article distributed under the terms and conditions of the Creative Commons Attribution (CC BY) license (http://creativecommons.org/licenses/by/4.0/). 